\title{
(2) OPEN ACCESS \\ Differential effects of epidermal growth factor receptor inhibitors in a single patient with neuropathic pain
}

\author{
Marte Grønlie Cameron, Christian Kersten
}

Center for Cancer Treatment, Southern Norway Hospital Trust, Kristiansand, Norway

Correspondence to Dr Marte Grønlie Cameron; Marte.Gronlie.Cameron@sshf.no

Accepted 10 March 2021

Check for updates

(c) BMJ Publishing Group Limited 2021. Re-use permitted under CC BY-NC. No commercial re-use. See rights and permissions. Published by BMJ.

To cite: Cameron MG, Kersten C. BMJ Case

Rep 2021:14:e239385.

doi:10.1136/bcr-2020-

239385

\section{SUMMARY}

Neuropathic pain (NP) represents an unmet medical need, where analgesic responses to different epidermal growth factor receptor inhibitors (EGFR-Is) have been described. The human EGFR family of receptors consists of four members (human epidermal growth factor receptor, HER 1-4), signalling via different homodimer and heterodimer combinations. A 52-year-old man was treated with the EGFR-I cetuximab in a trial of severe NP. Pain scores decreased dramatically after blinded cetuximab, but not after placebo. On pain recurrence after the trial, he was prescribed the oral EGFR-Is erlotinib, gefitinib, and lapatinib without relief. However, treatment with the pan-HER-inhibitor afatinib was effective. After 4 years on afatinib, pain control remains excellent with manageable side effects. This is the first reported observation of differential effects of EGFR-Is on NP in the same patient and the first report describing NP relief with afatinib. Further understanding of the underlying pathophysiology could lead to development of EGFR-Is specifically targeting NP.

\section{BACKGROUND}

Neuropathic pain (NP) is a clinical description of a pain state resulting from damage to the somatosensory nervous system. ${ }^{1}$ The pain is lancinating, electric shock-like and burning in character, associated with tingling and crawling sensations. Its severity, chronicity and the poor benefit to side effect ratio of pharmacotherapy for NP frequently lead to diminished physical and psychological functioning among sufferers. ${ }^{23}$ The prevalence of moderate to severe chronic NP in the western world is estimated to be at least $5 \%-8 \%$ and the global burden is escalating due to the ageing, growing population with rising prevalence of the chronic diseases causing NP, including postherpetic neuralgia, diabetic neuropathy, chemotherapy-induced peripheral neuropathy, lumbar radiculopathy and chronic postsurgical pain. ${ }^{45}$

The human epidermal growth factor receptor (EGFR) (human epidermal growth factor receptor, HER) family of cell surface receptors is made up of four members: ErbB1 (HER1, EGFR), ErbB2 (HER2), ErbB3 (HER3) and ErbB4 (HER4) (see figure 1). Each receptor consists of extracellular, transmembrane and intracellular domains. Binding of ligands to some of the extracellular domains leads to receptor dimerisation and conformational changes in the intracellular tyrosine kinases, resulting in receptor autophosphorylation and activation of downstream signal transduction pathways that regulate cellular functions. ${ }^{6}$ The concept of receptor dimerisation explains why HER2 can be active despite not having a known activating ligand and why HER 3 can bind ligands without activating its own kinase.

Both orally administered tyrosine kinase inhibitors (TKIs) and intravenously administered monoclonal antibodies have been developed to inhibit the EGFR. Several of these are approved and have been in use in oncology for more than a decade. ${ }^{8}$

Numerous TKIs, which target the intracellular domains of different transmembrane receptors have been under development for the treatment of malignancies. Some of them, such as erlotinib and gefitinib, are designed to specifically target the intracellular tyrosine kinase domain of the EGFR and are primarily used to treat non-small cell lung cancer. ${ }^{9}{ }^{10}$ Since cancers develop mutations in the tyrosine kinase domains of their EGFRs, second and third-generation oral EGFR-Is have been developed to overcome this.

Furthermore, TKIs designed to inhibit specific HER family heterodimers have also been developed. One example is lapatinib, a drug used to treat breast cancer, which binds to the EGFR/HER2 protein kinase domains. ${ }^{11}$ Afatinib inhibits all four HER kinases and is used in lung cancer.

The monoclonal antibody cetuximab inhibits functioning of the EGFR by targeting extracellular EGFR ligand binding domains. ${ }^{12}$ It has been licensed since 2004 to treat cancers of the head and neck and colorectal cancer. ${ }^{8}$

Side effects of EGFR-inhibitors are well described, based on their use in millions of cancer patients for over 15 years. Dry skin, acneiform rash and diarrhoea are most frequent side effects and feasibility of using EGFR-Is in the setting of NP presupposes supportive care and close follow-up. ${ }^{13}$

A serendipitous finding that cetuximab, independent of its anticancer effect, led to rapid relief of $\mathrm{NP}$ in a patient with malignant invasion of pelvic nerves sparked interest in the notion that it may also have therapeutic potential in NP. ${ }^{14}$ Since publication of the initial case, at least 84 additional clinical cases, including both benign and malignant causes of NP that were relieved by EGFR-Is have been reported. ${ }^{15-18}$ Analgesic responses to both antibodies (cetuximab and panitumumab) which work extracellularly and intracellular tyrosine kinase antagonists (erlotinib and gefitinib) are described. 


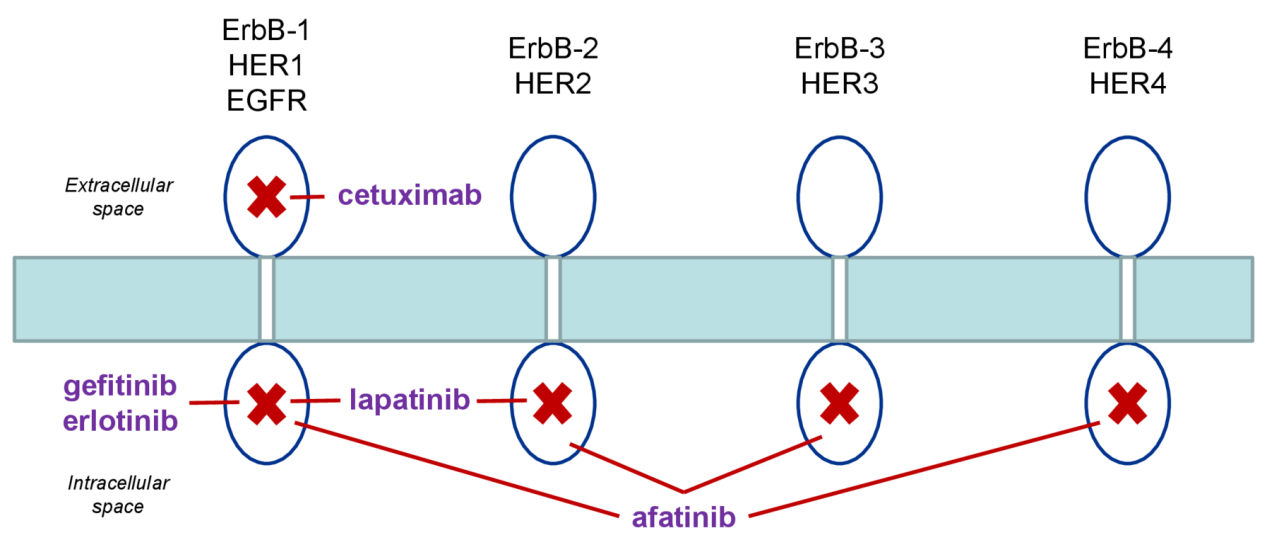

Figure 1 Simplistic representation of the four members of the human epidermal growth factor (HER) family of receptors traversing a cell membrane. The five epidermal growth factor receptor (EGFR)-inhibitors (and their corresponding targets) mentioned in this case report, are indicated in purple (and red). Ligand binding leads to receptor dimerisation and conformational changes in the intracellular tyrosine kinases, resulting in activation of downstream signal transduction. HER2 has no known ligand and HER3 no activating intracellular kinase, making them dependent on heterodimerisation for signalling.

Although the mechanism behind this phenomenon is not understood, it has clinically appeared to be a class effect. ${ }^{16} 18$ Chronic pain has recently been associated with mutations in the EGFR. ${ }^{19}$ Several papers describe a reduction of nocifensive behaviour in rodents with NP after administration of EGFRIs. ${ }^{19-21}$ One of these comprehensive studies convincingly demonstrates colocalisation of and increased molecular interaction between EGFR and HER2 on pain fibres in NP models as well as a synergistic role of combined EGFR and HER2 inhibition in NP relief. ${ }^{21}$ Another research group has suggested that EGFR/ HER4 heterodimers ${ }^{19}$ might be of importance. Together, clinical and preclinical findings point to the EGFR as a plausible target for treatment of NP.

Based on this, we performed a randomised, placebo-controlled double-blind proof-of-concept trial of cetuximab in patients with severe, treatment-refractory NP due to non-malignant compressed peripheral nerves $(n=7)$ or complex regional pain syndrome $(n=7){ }^{22}$ This so-called NoTOPain trial (NoTOPain:

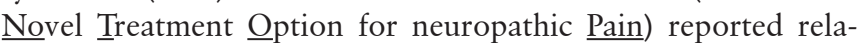
tively high response rates among treatment-resistant patients and the magnitude of pain relief seen in those patients who benefited was encouraging. ${ }^{22}$ Eight of the trial patients chose to continue with oral EGFR-Is after study completion. The current case report describes the clinical course of one of the trial participants during and for 48 months after the NoTOPain trial. This particular patient had convincing pain relief after cetuximab treatment within the placebo-controlled NoTOPain trial. Thereafter, he tried four different oral EGFR-Is, with mixed results.

\section{CASE PRESENTATION}

A 52-year-old previously healthy man with an 11-year history of recurrent, bilateral carpal tunnel syndrome presented with a 22-month history of severe NP in his right hand. The same condition was present on the left side, although to a lesser degree.

The patient had undergone multiple decompressive surgeries (two on the left side, three on the right) during the 11 years prior to the presentation. After each operation, he experienced symptom relief lasting from 6 to 9 months, followed by gradual recurrence. His last operation on his right wrist, 30 months prior to presentation, resulted in roughly 8 months of pain relief. Further surgical intervention was not recommended on pain recurrence. Various treatments were tried without satisfactory pain relief (see table 1). The pain was eventually deemed chronic and treatment refractory.

The patient described the pain as constantly present, with intensity usually ranging between 5 and 8 on a $0-10$ Numeric Rating Scale (NRS). The pain was located primarily distal to the wrists, in the area of median nerve innervation. Although there were both pain and sensory symptoms beyond this, both in the hand and lower forearm, he did not describe it as radiating. The pain was associated with numbness, tingling and prickling sensations. The most striking and clinically debilitating aspect of the patient's pain was the degree to which it was aggravated by cold, in terms of both cold allodynia and the effect of ambient temperature. He scored 22/38 on the PainDETECT questionnaire, indicating $>90 \%$ probability that the pain had a neuropathic component. $^{23}$

MRI of the right wrist revealed scar tissue in close proximity to the median nerve. Neurological examination found cold and light touch allodynia as well as decreased sensation distal to the wrist scars in both hands. These findings included but also went beyond the distribution of the median nerves. Findings were similar on both sides, but more severe on the right. Neurography confirmed damage to the right median nerve at the level of the carpal tunnel (the left side was not tested). Neurological examination was otherwise normal.

\section{Cetuximab}

The patient was referred to us for inclusion in the NoTOPain trial which tested EGFR-inhibition in patients with chronic, treatment-refractory $\mathrm{NP}^{22} \mathrm{He}$ was randomised to receive a single dose of blinded placebo first, followed by a single dose of

Table 1 Treatments tried for neuropathic pain

\begin{tabular}{lc}
\hline Medication/intervention & Analgesic effect \\
\hline Paracetamol & Partial effect \\
Ibuprofen & Partial effect \\
Weak opiate & Partial effect \\
TENS & Partial effect \\
Gabapentin/pregabalin & No effect \\
Acupuncture & No effect
\end{tabular}

TENS, transcutaneous electrical nerve stimulation. 


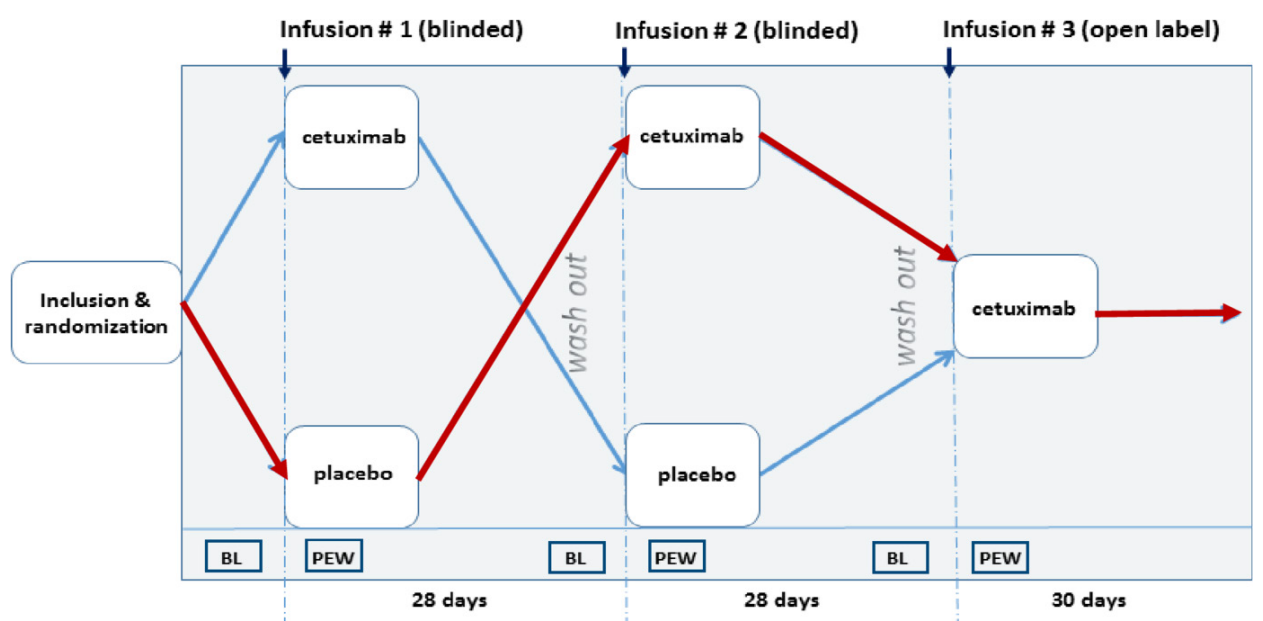

Figure 2 Schematic of NoTOPain trial design. Blinded single doses of intravenous cetuximab and placebo were administered in a cross-over fashion, followed by a single dose of open-label cetuximab. Red arrows indicate the patient's course through the trial. BL, baseline; NoTOPain trial, NoTOPain: Novel Treatment Option for neuropathic Pain; PEW, predefined evaluation window (3-7 days after each infusion).

blinded cetuximab and then one open-label cetuximab infusion (see figure 2).

The patient's pain scores (self-reported daily, on a 0-10 Numeric Rating Scale) showed a clinically significant decrease after blinded cetuximab, but not after placebo (see figure 3). Only the pain scores for the right hand (the most severely affected) were registered in the NoTOPain trial, although improvement was analogous on the left side.

The patient described the pain relief he experienced after cetuximab as having completely transformed his quality of life. Specifically, he was able to sleep right through the night, without being woken up by pain that he otherwise experienced several times each night when his hands were exposed to cold as they slipped out from under the bed covers. Being well-rested improved his concentration and capacity to work. Ability to tolerate cold meant that he no longer required warm gloves and could work outdoors in all types of weather. He was more flexible and functional in general, able to do spontaneously things he enjoyed with family and friends, no longer having to consider pain and disability. He was able to stop taking all other pain medications and no longer needed to use the transcutaneous electrical nerve stimulation apparatus at bedtime.

Mechanical allodynia was assessed during the NoTOPain trial using standardised nylon monofilaments before and after the patient was treated with blinded cetuximab. He was asked to identify the area on his right hand with maximum pain. The monofilaments, in order of increasing size and target force, were then sequentially applied there, and he was asked to indicate when the filament triggered a painful response (using a nonpainful area on the opposite side as a control). Just prior to the start of the cetuximab infusion, the patient scored 7 on the $0-10$ NRS for 'pain right now'. The first filament to provoke a pathological pain response at that time had a target force of $60 \mathrm{~g}$ (size 5.88). The test was repeated in the same place 2 hours after the cetuximab infusion, when his pain score was 1 on the same NRS (see figure 4). At that time, none of the monofilaments provoked

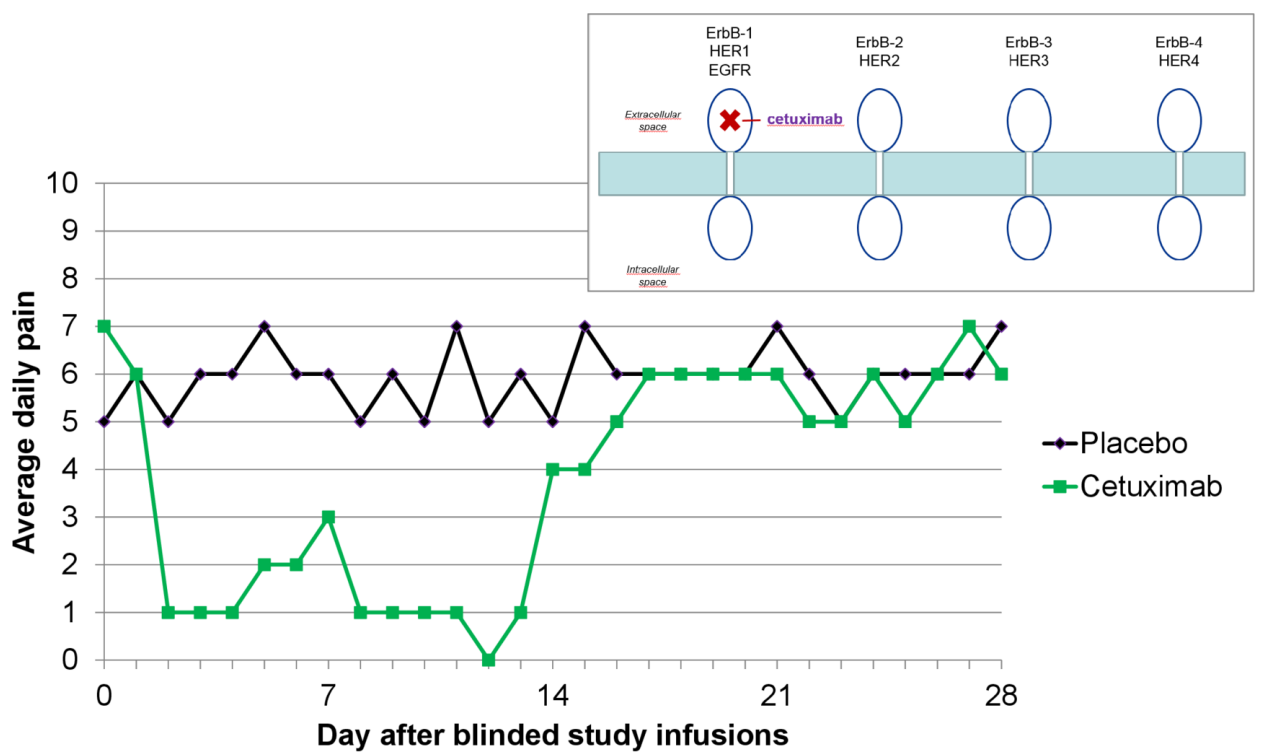

Figure 3 Patient-reported daily average pain scores (on a 0-10 Numeric Rating Scale) during the blinded infusions within the NoTOPain trial. Figure 1 is superimposed to indicate the site of epidermal growth factor receptor (EGFR)-inhibition by cetuximab. HER, human epidermal growth factor receptor; NoTOPain trial, NoTOPain: Novel Treatment Option for neuropathic Pain. 


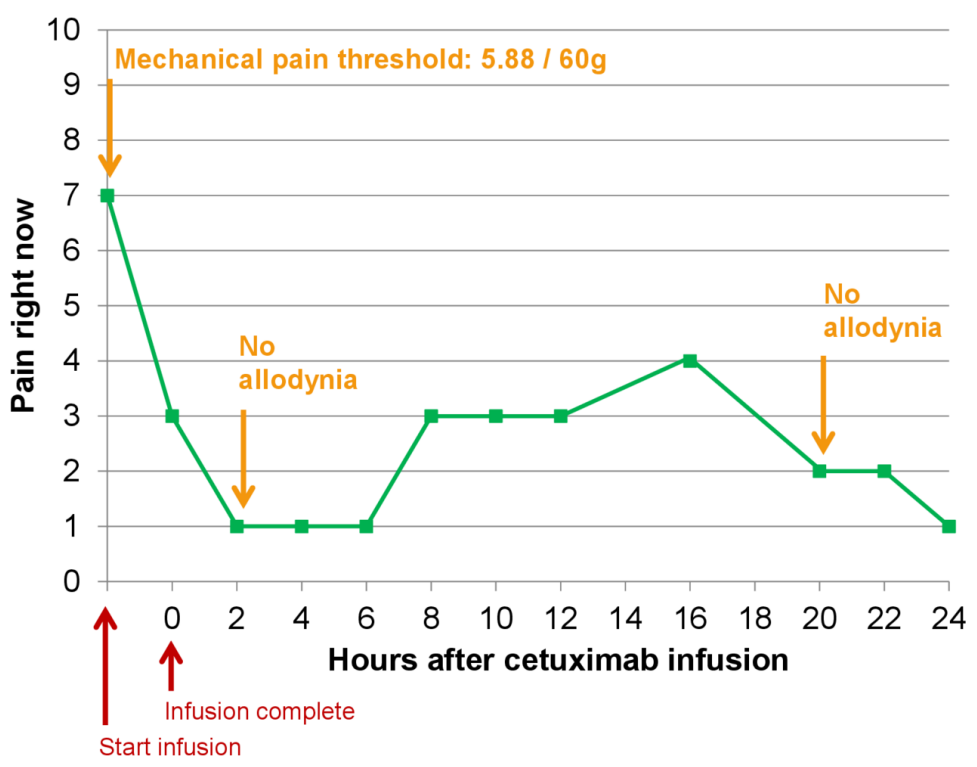

Figure 4 Patient-reported 'pain right now' scores on a 0-10 Numeric Rating Scale in the 24 hours after blinded cetuximab infusion during the NoTOPain trial. Yellow arrows indicate timing of assessment of mechanical allodynia. NoTOPain trial, NoTOPain: Novel Treatment Option for neuropathic Pain.

a pathological pain response (the largest filament tested having target force $300 \mathrm{~g}$ (size 6.65)). The test was repeated again the following day, with a pain score of 2 and again allodynia was not provoked by any of the monofilaments.

\section{Erlotinib}

The patient experienced near-complete pain-relief after one of the two blinded study infusions which at study closure was revealed to be cetuximab. The open-label cetuximab infusion elicited the same response. He was, therefore, prescribed the oral EGFR-inhibitor erlotinib $150 \mathrm{mg}$ daily, which he started taking once pain recurrence was well-established after his last cetuximab infusion. The patient described partial, transient improvement in NP after the first dose of erlotinib but felt that it never measured up to the dramatic improvement that he had experienced after the two intravenous cetuximab infusions in the trial.

The question of whether lack of efficacy of erlotinib could be a dosing question was raised so after 2 weeks taking $150 \mathrm{mg}$ he increased to $300 \mathrm{mg}$ daily. He took $300 \mathrm{mg}$ daily for a total of 6 days but stopped because he was then convinced that erlotinib in fact had no effect at all, that is, he reported that the pain was back to the levels he had before start of the trial.

\section{Gefitinib}

After a further month of pain, treatment with the oral EGFRinhibitor gefitinib was started. He took $250 \mathrm{mg}$ gefitinib daily for 3 weeks without any change in NP severity.

\section{Afatinib}

Having failed both oral EGFR-Is that had shown benefit in several patients before, ${ }^{15} 16$ afatinib was prescribed, based on the hypotheses that a germline EGFR mutation may cause resistance to erlotinib and gefitinib, or that a broader inhibition (afatinib is a pan-HER inhibitor) may reproduce the effect he had experienced after the two cetuximab infusions in the NoTOPain trial. Therefore, 6 months after his last dose of cetuximab in the trial, the patient was prescribed afatinib $40 \mathrm{mg}$ daily. He began noticing improvement in his NP on day 4 of this treatment.
There was a gradual decrease in pain scores over the subsequent 2-3 weeks until a new plateau was reached (see figure 5).

Again, pain relief was accompanied by improved function and enjoyment of life, as indicated by Brief Pain Inventory scores (see table 2).

After approximately 3 months, the patient's afatinib dose was reduced to $20 \mathrm{mg}$ daily in order to test if that was a sufficient dose for pain control. The patient's impression is that afatinib is not fully as effective as he remembers intravenous cetuximab but that $20 \mathrm{mg}$ daily of afatinib is virtually as effective as $40 \mathrm{mg}$, with lesser side effects. During the treatment breaks, his pain typically recurs after 3 days.

Afatinib may inhibit EGFRs with mutations that render them resistant to erlotinib and gefitinib. The patient's blood was therefore analysed for germline EGFR mutations with allelespecific PCR (Cobas EGFR mutation test v2, Roche), covering 42 different mutations in exons 18, 19, 20 and 21. None of these mutations were found.

\section{Lapatinib}

A year after starting afatinib, the patient was offered a trial of the HER1/HER2 inhibitor lapatinib $1250 \mathrm{mg}$ daily in an attempt to reduce gastrointestinal and cutaneous side effects. He took this treatment for 7 days without noticing any improvement in his $\mathrm{NP}$ and therefore reverted to afatinib.

\section{Side effects}

The patient has intermittently taken tetracycline $500 \mathrm{mg}$ two times per day to treat acneiform rash while under treatment with the various EGFR-Is. He has had maximum grade 2 dry skin and mucositis. His most bothersome side effect has been grade 2 diarrhoea, for which he has taken loperamide as needed, with partial effect.

\section{OUTCOME AND FOLLOW-UP}

Currently, 4 years after starting afatinib, he characterises his pain as completely gone as long as he takes $25 \mathrm{mg}$ of afatinib daily. He has found that this dose gives him an effect to side-effect balance that results in the best possible quality of life. Based on 


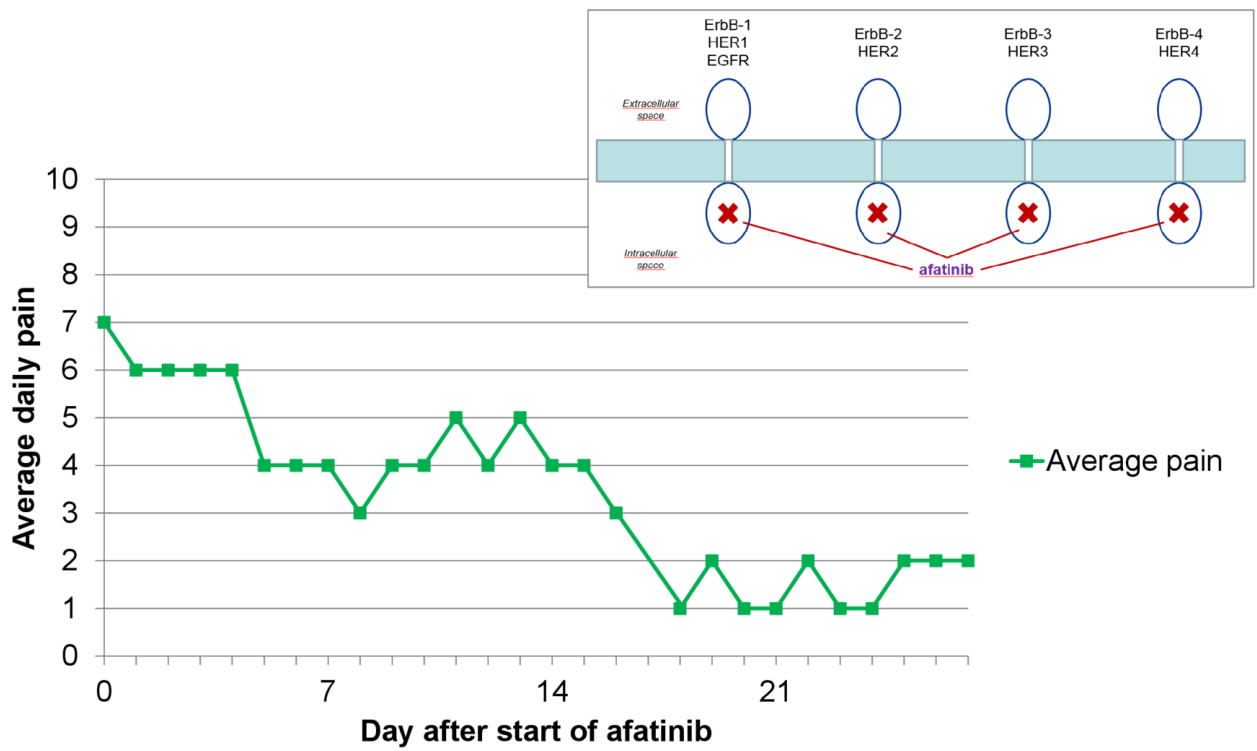

Figure 5 Patient-reported daily average pain scores (on a 0-10 Numeric Rating Scale) after he began taking afatinib $40 \mathrm{mg}$ daily. Figure 1 is superimposed to indicate the site of human epidermal growth factor receptor (HER)-inhibition by afatinib. EGFR, epidermal growth factor receptor.

the experience with this case, an additional five patients who were using erlotinib $(n=4)$ or gefitinib $(n=1)$ have tried afatinib and reported better effect, tolerability or both.

\section{DISCUSSION}

This is the first time different effects of EGFR-Is on NP have been described in the same patient. This is also the first report describing NP relief with afatinib.

We have previously reported NP relief by the intravenous EGFR-Is cetuximab and panitumumab ${ }^{14-16} 18$ and the oral TKIs erlotinib and gefitinib. ${ }^{151618}$ However, in all previously reported cases, pain responses were similar across EGFR-Is and in accordance with their established pharmacokinetics.

This case report resembles an $\mathrm{N}=1$ study in that the same patient received four different EGFR-Is in series, with pain recurrence both between the different EGFR-Is tested and during treatment breaks between doses of cetuximab and afatinib. In contrast to a planned $\mathrm{N}=1$ study, however, this patient's treatment course was guided not by a protocol, but by clinical decisions based on responses to the various EGFR-Is he was given along the way.

Table 2 Patient-reported functional scores during the 24 hours prior to starting afatinib and again after having taken afatinib $40 \mathrm{mg}$ daily for 4 weeks

\begin{tabular}{lcc}
\hline BPI function & 0-10 score before afatinib & $\begin{array}{l}0-10 \text { score } \\
\text { 4 weeks after afatinib }\end{array}$ \\
\hline General activity & 6 & 3 \\
Mood & 6 & 5 \\
Walking ability & 0 & 0 \\
Normal work & 5 & 3 \\
Relationship with others & 5 & 2 \\
Sleep & 9 & 1 \\
Enjoyment of life & 7 & 2 \\
Total & 38 & 16 \\
\hline
\end{tabular}

Scoring is done on $0-10$ Numeric Rating Scales where higher scores indicate worse function.

$\mathrm{BPI}$, brief pain inventory.
A strength of this case report is the prospective capture of patient-reported outcomes of pain and function. Although not generalisable, the observations support the notion of EGFR-I as a viable treatment option for NP, particularly since this otherwise treatment-refractory patient has continued to take afatinib for 4 years (to date), in order to maintain a better quality of life.

The fact that a patient whose NP responded to cetuximab but not to erlotinib, gefitinib or lapatinib, responded to afatinib is hypothesis generating.

\section{Biological hypotheses}

Better understanding of the biological mechanisms underlying the differential effects described in this case could potentially unlock a key to developing EGFR-Is specifically targeting NP, with a conceivably larger therapeutic window.

In principle, these biological mechanisms could be located at the level of the (1) EGFR ligands, (2) transmembrane receptors or (3) downstream of the receptors. Physiological or pathological mechanisms of resistance could also occur on all these levels. The fact that afatinib inhibits all HER receptors may indicate a broader effect on more extracellular ligands and/or on more intracellular downstream signalling pathways. However, it is important to note that several mechanisms could occur simultaneously, change over time and, importantly, be context dependent.

\section{EGFR ligands}

Seven EGFR ligands have been identified, ${ }^{24}$ including epiregulin which is involved in the generation of NP. ${ }^{1925}$ The way each of these ligands interact with the EGFR determines their differential effects. ${ }^{26}$ Non-EGFR HER ligands such as neuregulins are also implicated in the pathophysiology of NP. ${ }^{27} 28$ Thus, one can speculate that different HER ligands are involved in the pathophysiologies of the individual NP patients. If so, this would also be mirrored by their respective susceptibilities to inhibition by the different EGFR-Is. 
Transmembrane receptors

If this patient's NP is driven by signalling via an EGFR heterodimer, it follows that extracellular binding of cetuximab to the EGFR would inhibit heterodimer formation regardless of which of the other HERs it is coupling with. ${ }^{19}$ This could explain why erlotinib and gefitinib, both highly selective EGFR-Is with an intracellular mode of action, were not effective, while the panHER-inhibitor afatinib is. This points to an additive or even synergistic effect of certain HER-receptor inhibitor combinations, which has been hypothesised ${ }^{19} 29$ and shown in rodent models. ${ }^{21}$ As indicated in figure 1, afatinib binds to the EGFR, HER 2 and HER $4 .{ }^{30}$ By doing so, it irreversibly inhibits signalling of the entire HER family of receptors, including HER3, to which it does not bind directly.

Lapatinib, a dual inhibitor of the EGFR and HER2 receptors ${ }^{31}$ has been shown to inhibit pain in rodent models. ${ }^{19}{ }^{21}$ It has, however, also been shown to strengthen the inactive monomeric form of the EGFR, thereby preventing its dimerisation. ${ }^{32}$ The fact that the patient did not experience pain relief while taking lapatinib, thus raises the question as to the extent to which the EGFR or relevant heterodimer, was being inhibited by that drug, since recent preclinical findings demonstrate a strong synergistic analgesic effect when both EGF and HER2 receptors are inhibited. ${ }^{21}$ Interestingly, the relative half maximal inhibitory concentrations $_{50}$ of these drugs to inhibit the EGF and HER2 receptors favours EGFR-inhibition by afatinib compared with lapatinib, which may explain the observed differences in pain responses in our patient. ${ }^{2933}$

\section{Intracellular downstream signalling}

Net outcome of EGFR-I depends on cellular context. ${ }^{34}$ A metaanalysis of 20 individual studies comprising 346 microarrays reported that different EGFR-Is had overlapping but quite distinct transcriptional results in target cells. ${ }^{34}$ To our knowledge, no such studies have been performed on damaged peripheral nerve cells. This could be relevant in our patient.

\section{Physiological or pathological mechanisms of resistance}

Many different germline and acquired resistance mechanisms against EGFR-I have been described in the context of cancer therapy. ${ }^{35} 36$ Such mechanisms could potentially also play a role in the differential pain relief observed in our patient.

The patient could in theory be a carrier of:

1. A germline mutation known to be targeted by afatinib (the patient has tested negative for these).

2. A rare germline mutation, where afatinib works. ${ }^{37}$

3. Certain single-nucleotide polymorphisms (SNP) involved in pain syndromes, ${ }^{19}$ where afatinib is effective but undocumented.

4. An acquired somatic mutation or SNP in the peripheral sensory nerves that involves mechanisms 1-3.

Other acquired resistance mechanisms against EGFR-I include HER2 upregulation, activation of bypass signalling and noncoding RNA. ${ }^{35}$ Our patient could in theory have developed one or more such resistance mechanisms. However, of the more than 85 patients that we know have been treated with EGFR-Is for $\mathrm{NP}^{18}$ acquired resistance against EGFR-inhibition has been observed only once, after one and a half years of continuous treatment with EGFR-I (unpublished observation).

\section{Psychological hypothesis}

The patient experienced the same magnitude of near-immediate pain relief after both blinded and open-label cetuximab infusions within the NoTOPain trial. ${ }^{22}$ His pain was not relieved by matched placebo (see figure 3). The rapid, although transient pain relief that began after a single dose of erlotinib was most likely a placebo response. Erlotinib reaches maximum plasma concentration between 0.5 and 4 hours after ingestion and the terminal half-life is $1.5-3$ hours. ${ }^{38}$ Accordingly, it is not impossible to experience rapid pain relief as described. However, experience in other patients indicates a time to relevant treatment effect of several days when using oral EGFR-Is. ${ }^{15} 16$ The long-lasting effect of afatinib argues against a placebo effect, particularly when the two preceding TKIs (erlotinib and gefitinib) and later lapatinib, were ineffectual.

\section{Limitations}

There are obvious limitations to the validity and generalisability of subjective findings reported by a single patient. However, the value of this case lies in its hypothesis-generating role. Lack of translational elements (other than a germline mutation analysis of the EGFR) hinder further validation of the hypotheses presented, including the relevance of heterodimer signalling. Further studies in animal models should explore these avenues, which could subsequently be verified in human series.

\section{Future perspectives}

Not all EGFR-Is are created equal. We have previously proposed a class effect for EGFR-Is in the treatment of NP and by and large this still seems valid. However, this particular case may indicate that there are nuances of the EGFR/NP association that may open up avenues for drug development.

Although common, cutaneous side effects of EGFR-Is are usually mild and acceptable for patients treated for severe and otherwise treatment resistant NP. ${ }^{22}$ However, skin reactions may hamper long-term use of EGFR-Is in patients with less severe NP. If a relationship exists between different HER family heterodimers and toxicity ${ }^{39}$ and/or therapeutic effect of anti-EGFRdirected therapy against NP, this could potentially lead to more targeted therapy, reducing toxicity while maintaining or even increasing the analgesic effect.

\section{Learning points}

- Several epidermal growth factor receptor inhibitors (EGFR-Is) have previously been reported to relieve neuropathic pain (NP), indicating a possible class effect.

- NP in this case responded differently to different EGFR-Is.

- This case illustrates for the first time that the EGFR-I afatinib can relieve NP.

- Novel observations in this case are supported by preclinical research and indicate a possibility to develop an EGFR-I with an optimal profile to specifically target NP.

Contributors Both authors (MGC and CK) contributed to treatment of the patient, collection and interpretation of clinical data and writing the manuscript.

Funding This study was funded by Helse Sør-Øst RHF (2016130).

Competing interests The authors have filed an international patent application according to the Patent Cooperation Treaty, for targeting the EGFR in neurological disorders. CK has, since May 2020 been employed by Roche Norway AS which owns erlotinib.

Patient consent for publication Obtained

Provenance and peer review Not commissioned; externally peer reviewed.

Open access This is an open access article distributed in accordance with the Creative Commons Attribution Non Commercial (CC BY-NC 4.0) license, which permits others to distribute, remix, adapt, build upon this work non-commercially, 
and license their derivative works on different terms, provided the original work is properly cited and the use is non-commercial. See: http://creativecommons.org/ licenses/by-nc/4.0/.

\section{REFERENCES}

1 Finnerup NB, Haroutounian S, Kamerman P, et al. Neuropathic pain: an updated grading system for research and clinical practice. Pain 2016;157:1599-606.

2 Finnerup NB, Attal N, Haroutounian S, et al. Pharmacotherapy for neuropathic pain in adults: a systematic review and meta-analysis. Lancet Neurol 2015;14:162-73.

3 Chaparro LE, Wiffen PJ, Moore RA, et al. Combination pharmacotherapy for the treatment of neuropathic pain in adults. Cochrane Database Syst Rev 2012;7:CD008943.

4 Yawn BP, Wollan PC, Weingarten TN, et al. The prevalence of neuropathic pain: clinical evaluation compared with screening tools in a community population. Pain Med 2009;10:586-93.

5 Attal N, Lanteri-Minet M, Laurent B, et al. The specific disease burden of neuropathic pain: results of a French nationwide survey. Pain 2011;152:2836-43.

6 Wieduwilt MJ, Moasser MM. The epidermal growth factor receptor family: biology driving targeted therapeutics. Cell Mol Life Sci 2008;65:1566-84.

7 Roskoski R. Small molecule inhibitors targeting the EGFR/ErbB family of proteintyrosine kinases in human cancers. Pharmacol Res 2019;139:395-411.

8 Roskoski R. The ErbB/HER family of protein-tyrosine kinases and cancer. Pharmacol Res 2014;79:34-74.

9 EMA. Ema summary of product characteristics for erlotinib, 2018

10 EMA. Ema summary of product characteristics for gefitinib, 2018.

11 EMA. Lapatinib summary of product characteristics, 2018.

12 EMA. Cetuximab_Summary of product characteristics, 2017. Available: http:// www.medicines.org.uk/emc/medicine/19595/SPC/Erbitux+5mg+ml+solution+for+ infusion\#PHARMACOKINETIC PROPS

13 Melosky B, Leighl NB, Rothenstein J, et al. Management of EGFR TKi-induced dermatologic adverse events. Curr Oncol 2015:22:123-32.

14 Kersten C, Cameron MG. Cetuximab alleviates neuropathic pain despite tumour progression. BMJ Case Rep 2012;2012. doi:10.1136/bcr.12.2011.5374

15 Kersten C, Cameron M, Mjaaland S. Epithelial growth factor receptor (EGFR)inhibition for relief of neuropathic pain-A case series. Scandinavian Journal of Pain 2013;2013:3-7

16 Kersten C, Cameron MG, Laird B, et al. Epidermal growth factor receptor-inhibition (EGFR-I) in the treatment of neuropathic pain. Br J Anaesth 2015;115:761-7.

17 Shu Yuasa MK, Furuta R, Hibi S. A case of sigmoid colon cancer in which somatic pain was rapidly alleviated after panitumumab administration despite tumor progression. Journal of Analytical Oncology 2016;5:38-41.

18 Cameron MK C. EGFR-inhibition in neuropathic pain - A synthesis of experience With EGFR-Is in different neuropathic pain entities -. NeuPSIG 2019 London: 7th International Congress on Neuropathic Pain, 2019: PS 84.

19 Martin LJ, Smith SB, Khoutorsky A, et al. Epiregulin and EGFR interactions are involved in pain processing. J Clin Invest 2017:127:3353-66.
20 Wang S, Liu S, Xu L, et al. The upregulation of EGFR in the dorsal root ganglion contributes to chronic compression of dorsal root ganglions-induced neuropathic pain in rats. Mol Pain 2019;15:1744806919857297.

21 Mitchell R, Mikolajczak M, Kersten C, et al. ErbB1-dependent signalling and vesicular trafficking in primary afferent nociceptors associated with hypersensitivity in neuropathic pain. Neurobiol Dis 2020;142:104961.

22 Kersten C, Cameron MG, Bailey AG, et al. Relief of neuropathic pain through epidermal growth factor receptor inhibition: a randomized proof-of-concept trial. Pain Med 2019;20:2495-505.

23 Freynhagen R, Baron R, Gockel U, et al. painDETECT: a new screening questionnaire to identify neuropathic components in patients with back pain. Curr Med Res Opin 2006;22:1911-20.

24 Harris RC, Chung E, Coffey RJ. Egf receptor ligands. Exp Cell Res 2003;284:2-13.

25 Kongstorp M, Schjølberg T, Jacobsen DP, et al. Epiregulin is released from intervertebral disks and induces spontaneous activity in pain pathways. Pain Rep 2019;4:e718.

26 Roepstorff K, Grandal MV, Henriksen L, et al. Differential effects of EGFR ligands on endocytic sorting of the receptor. Traffic 2009;10:1115-27.

27 Linggi B, Carpenter G. Erbb receptors: new insights on mechanisms and biology. Trends Cell Biol 2006;16:649-56.

28 Dai D-W, Xu Z, Chen X, et al. Distinct roles of neuregulin in different models of neuropathic pain. Neurol Sci 2014;35:531-6.

29 Cameron MKC, Kersten C. Differential effects of epidermal growth factor receptor inhibitors in a single case of neuropathic pain. EFIC Congress 2019 - Pain in Europe XI, 2019.

30 Dungo RT, Keating GM. Afatinib: first global approval. Drugs 2013;73:1503-15.

31 Medina PJ, Goodin S. Lapatinib: a dual inhibitor of human epidermal growth factor receptor tyrosine kinases. Clin Ther 2008:30:1426-47.

32 Björkelund H, Gedda L, Malmqvist M, et al. Resolving the EGF-EGFR interaction characteristics through a multiple-temperature, multiple-inhibitor, real-time interaction analysis approach. Mol Clin Oncol 2013;1:343-52.

33 , 2020. Available: https://www.selleckchem.com/

34 Blumenberg M. Differential transcriptional effects of EGFR inhibitors. PLoS One 2014;9:e102466.

35 Zhang K, Yuan Q. Current mechanism of acquired resistance to epidermal growth factor receptor-tyrosine kinase inhibitors and updated therapy strategies in human nonsmall cell lung cancer. J Cancer Res Ther 2016:12:131-7.

36 Mukohara T, Engelman JA, Hanna NH, et al. Differential effects of gefitinib and cetuximab on non-small-cell lung cancers bearing epidermal growth factor receptor mutations. J Nat/ Cancer Inst 2005;97:1185-94.

37 Yang JC-H, Schuler M, Popat S, et al. Afatinib for the treatment of NSCLC harboring uncommon EGFR mutations: a database of 693 cases. J Thorac Oncol 2020;15:803-15.

38 EMA. Erlotinib documents by European medicines Agency, 2019. Available: https:// wwwemaeuropaeu/en/medicines/human/EPAR/tarceva

39 Laux I, Jain A, Singh S, et al. Epidermal growth factor receptor dimerization status determines skin toxicity to HER-kinase targeted therapies. $\mathrm{Br} J$ Cancer 2006;94:85-92.

Copyright 2021 BMJ Publishing Group. All rights reserved. For permission to reuse any of this content visit https://www.bmi.com/company/products-services/rights-and-licensing/permissions/

BMJ Case Report Fellows may re-use this article for personal use and teaching without any further permission.

Become a Fellow of BMJ Case Reports today and you can:

- Submit as many cases as you like

Enjoy fast sympathetic peer review and rapid publication of accepted articles

Access all the published articles

Re-use any of the published material for personal use and teaching without further permission

Customer Service

If you have any further queries about your subscription, please contact our customer services team on +44 (0) 2071111105 or via email at support@bmj.com.

Visit casereports.bmi.com for more articles like this and to become a Fellow 